\title{
Prevalencia de enfermedades cardiovasculares en el Hospital Nacional Dos de Mayo de Perú
}

\section{Prevalence of cardiovascular diseases in the Dos de Mayo National Hospital in Peru}

\author{
Diego Chambergo-Michilot ${ }^{1,2 *}$, Bruno Velit-Rios ${ }^{1}$ y Angel Cueva-Parra ${ }^{3}$ \\ ${ }^{1}$ Escuela de Medicina Humana, Facultad de Ciencias de la Salud, Universidad Cientifica del Sur, Lima; ${ }^{2}$ Tau-RELAPED Group, Trujillo; ${ }^{3}$ Servicio de \\ Cardiología, Hospital Nacional Dos de Mayo, Lima. Perú
}

\begin{abstract}
Resumen
Objetivo: Conocer la prevalencia de las enfermedades cardíacas y sus comorbilidades en todos los pacientes de un servicio de cardiología de un hospital peruano. Material y métodos: Se realizó un estudio observacional descriptivo en un hospital peruano de tercer nivel (Hospital Nacional Dos de Mayo, Lima, Perú) entre enero del 2016 y diciembre del 2017. Resultados: Se incluyó 446 pacientes que ingresaron al servicio. Las enfermedades cardíacas más frecuentes fueron la falla cardíaca (51.57\%), cardiopatías congénitas (23.99\%), fibrilación auricular (17.49\%) e infarto miocárdico agudo (14.57\%). Las comorbilidades más frecuentes fueron la hipertensión arterial (7.62\%), enfermedad renal crónica (5.38\%) y diabetes mellitus (2.02\%). Conclusiones: Este estudio reporta las enfermedades y comorbilidades cardiovasculares en un servicio de cardiología. Los resultados servirán para el planteamiento de la muestra de otros estudios, y podrían ser de utilidad para los administradores en salud, ya que tendrán evidencia para priorizar los recursos para las enfermedades más frecuentes.
\end{abstract}

Palabras clave: Prevalencia. Insuficiencia cardíaca. Hospital. Perú.

\section{Abstract}

Objective: To know the prevalence of heart diseases and their comorbidities in all patients of a cardiology service from a Peruvian hospital. Material and methods: A descriptive observational study was conducted in a third level Peruvian hospital (Dos de Mayo National Hospital, Lima, Peru) between January 2016 and December 2017. Results: We included 446 patients who entered the service. The most frequent heart diseases were heart failure (51.57\%), congenital heart defects (23.99\%), atrial fibrillation (17.49\%) and acute myocardial infarction (14.57\%). The most frequent comorbidities were arterial hypertension (7.62\%), chronic kidney disease (5.38\%) and diabetes mellitus (2.02\%). Conclusions: This study reports heart diseases and comorbidities in a cardiology service. The results will serve for the approach of the sample of other studies, and could be useful for health administrators, since they will have evidence to prioritize resources for the most frequent diseases.

Key words: Prevalence. Heart failure. Hospitals. Peru.

Correspondencia:

*Diego Chambergo-Michilot

E-mail: diegochambergomichilot@ hotmail.com
Disponible en internet: $26-10-2020$ Rev Mex Angiol. 2020;48(3):84-89 www.RMAngiologia.com 0377-4740/@ 2020 Sociedad Mexicana de Angiología y Cirugía Vascular y Endovascular, A.C. Publicado por Permanyer. Este es un artículo open access bajo la licencia CC BY-NC-ND license (http://creativecommons.org/licenses/by-nc-nd/4.0/). 


\section{Introducción}

Las enfermedades cardíacas forman parte de las primeras causas de muerte en Latinoamérica y en el mundo, ${ }^{1,2}$, además son causas importantes de discapacidad ${ }^{1,3}$.

Se han reportado diferentes cifras epidemiológicas en países latinoamericanos, no obstante, la mayoría de estudios solo informan de cuáles son las enfermedades cardíacas más frecuentes en la población general $^{2,4,5}$, dejando de lado la descripción de cuáles de ellas generan la mayor cantidad de ingresos a los servicios hospitalarios. Los estudios de prevalencia de enfermedades intrahospitalarias son relevantes porque los resultados sobre las enfermedades con las mayores cifras servirán para que los administradores de salud puedan priorizarlas en relación con el uso de recursos económicos.

Perú, un país latinoamericano, presenta una población con prevalencia considerable de factores de riesgo cardiovascular. Un estudio de representatividad nacional reportó que la prevalencia de hipertensión, obesidad y diabetes fue del 20.1, 22.7 y $3.6 \%$, respectivamente, en el $2018^{6}$. Asimismo, otro estudio multicéntrico en Perú reveló que el $31.8 \%$ presentó pobre salud cardiovascular, que es un puntaje basado en factores y comportamientos cardiovasculares saludables ${ }^{7}$. Tomando en cuenta estos datos de la población general, podemos hipotetizar que los ingresos a hospitalización por causas cardíacas son elevados. No obstante, existen pocos estudios que hayan reportado la prevalencia de estas enfermedades a nivel hospitalario.

Ríos-Navarro, et al. ${ }^{8}$ caracterizaron clínicamente a los pacientes con infarto miocárdico de un hospital peruano, reportando comorbilidades, clase funcional, fracción de eyección ventricular, creatinina, entre otras variables. De la misma forma, Pariona, et al. ${ }^{9}$ reportaron las características clínicas de pacientes con falla cardíaca en el mismo hospital. A pesar de la utilidad de estos estudios, la importancia de sus resultados se limita a estas enfermedades. Por otro lado, Aronow, et al. ${ }^{10}$ reportaron las cifras de enfermedades cardíacas en un centro de atención del adulto mayor. Los autores reportaron que la falla cardíaca, enfermedad arterial periférica y enfermedad coronaria fueron las patologías más importantes. Esta información será útil para la dirección del uso de recursos a la atención de estas enfermedades, además será útil para la prevención terciaria de estas enfermedades.

Consecuentemente, nuestro objetivo fue describir las características clínicas de los pacientes del Servicio de Cardiología del Hospital Nacional Dos de Mayo, Lima,
Perú, un centro de salud de referencia en Perú. Este hospital fue el primer centro hospitalario público fundado en el país, y recibe pacientes de todos los niveles socioeconómicos referidos de todas las ciudades.

\section{Materiales y métodos}

\section{Diseño de estudio}

Se realizó un estudio observacional descriptivo.

\section{Población y muestra}

La población accesible fueron todos los pacientes que ingresaron por emergencias o de forma programada para alguna intervención diagnóstica y/o terapéutica al Servicio de Cardiología del Hospital Nacional Dos de Mayo, Lima, Perú, entre enero del 2016 y diciembre del 2017. La información se obtuvo de las historias clínicas de los pacientes.

No se realizó cálculo de muestra ni muestreo porque se evaluó a toda la población accesible. No se realizó exclusión por edad ni por patología específica.

Para ampliar el panorama general, describimos que este hospital presenta una cantidad total de 586 camas $^{11}$. Asimismo, en el 2017 (periodo de estudio), la cantidad de pacientes atendidos fue $91.393^{12}$.

\section{Variables}

No hubo variable dependiente 0 independiente por la naturaleza del objetivo del estudio.

Se recolectó la edad (años), si es adulto mayor (sí o no), sexo (masculino o femenino), comorbilidades y enfermedades cardíacas. Debido al gran número de comorbilidades y enfermedades, estas fueron recolectadas a través de las variables "comorbilidades» y «enfermedades cardíacas», para luego ser convertidas en variables dummy (sí o no) en relación con cada comorbilidad y enfermedad cardíaca.

El diagnóstico de las enfermedades fue realizado utilizando las correspondientes guías de práctica clínica. Por ejemplo, la diabetes fue diagnosticada utilizando los criterios de la American Diabetes Association ${ }^{13}$, y la falla cardíaca fue diagnosticada utilizando la guía más actual de la European Society of Cardiology ${ }^{14}$.

\section{Análisis}

La base fue digitalizada en Microsoft Excel, y luego fue exportada al paquete estadístico STATA v.14 


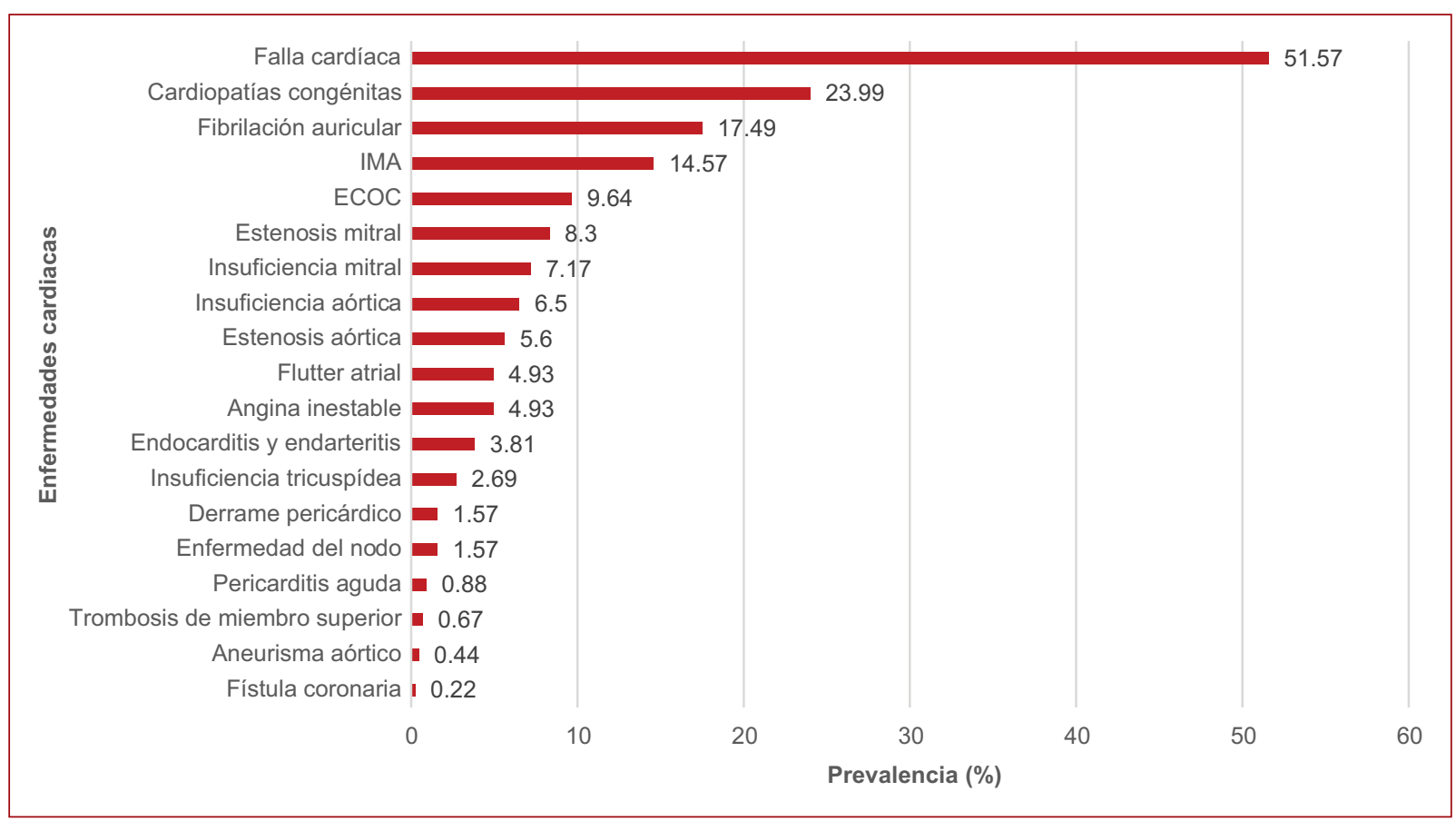

Figura 1. Prevalencia de enfermedades cardiacas en el Servicio de Cardiología del Hospital Nacional Dos de Mayo $(n=446)$. ECOC: enfermedad coronaria obstructiva crónica; IMA: infarto miocárdico agudo.

(College Station, TX: StataCorp LLC) para el análisis. Las variables cualitativas se expresaron en frecuencia, porcentaje e intervalo de confianza al $95 \%$; las variables continuas se expresaron en media \pm desviación estándar (DE) o mediana (rango intercuartílico) según su normalidad, que fue evaluada por la prueba de Kolmogorov-Smirnov.

El gráfico presentado fue realizado mediante Microsoft Excel.

\section{Resultados}

Se incluyó 446 pacientes que ingresaron al servicio. La media de edad fue 50.54 años (DE: 25.44), y 6 de cada 10 pacientes fueron hombres $(60.76 \%)$. Además, cerca de la mitad fueron adultos mayores (45.52\%). Las enfermedades cardíacas más prevalentes fueron la falla cardíaca $(51.57 \%)$, cardiopatías congénitas $(23.99 \%)$, fibrilación auricular (17.49\%) e infarto miocárdico agudo (14.57\%). Respecto al infarto, la mayoría (92.31\%) presentó elevación del segmento ST. Dentro de los pacientes con falla cardíaca, la falla descompensada significó el $13.04 \%$. Las patologías más raras en el servicio fueron trombosis de miembro superior $(0.67 \%)$, aneurisma aórtico $(0.44 \%)$ y fístula coronaria $(0.22 \%)$
Tabla 1. Comorbilidades crónicas en el Servicio de Cardiología del Hospital Nacional Dos de Mayo, Lima, Perú $(n=446)$

\begin{tabular}{|l|c|c|c|}
\hline Variables & $\mathbf{n}$ & $\%$ & IC $95 \%$ \\
\hline Edad (media \pm DE) & $50.54 \pm 25.44$ & - & - \\
\hline $\begin{array}{l}\text { Adultos mayores } \\
\quad \text { No }\end{array}$ & 243 & 54.48 & $49.82-59.07$ \\
\hline Sí & 203 & 45.52 & $40.93-50.18$ \\
\hline Sexo & & & \\
$\quad$ Masculino & 271 & 60.76 & $56.13-65.21$ \\
\hline Femenino & 175 & 39.24 & $34.79-43.86$ \\
\hline Comorbilidades & & & \\
$\quad$ HTA & 34 & 7.62 & $5.49-10.49$ \\
ERC & 24 & 5.38 & $3.63-7.91$ \\
Diabetes mellitus & 9 & 2.02 & $1.05-3.84$ \\
DCV & 3 & 0.67 & $0.22-2.07$ \\
Hepatopatía crónica & 1 & 0.22 & $0.03-1.16$ \\
Hipotiroidismo & 1 & 0.22 & $0.03-1.16$ \\
EPOC & 1 & 0.22 & $0.03-1.16$ \\
Infección por VIH & 1 & 0.22 & $0.03-1.16$ \\
\hline
\end{tabular}

DCV: desorden cerebrovascular; DE: desviación estándar; EPOC: enfermedad pulmonar obstructiva crónica; ERC: enfermedad renal crónica; HTA: hipertensión arterial; IC 95\%: intervalo de confianza al 95\%; VIH: virus de la inmunodeficiencia humana.

(Fig. 1). Por último, las comorbilidades más frecuentes fueron hipertensión arterial (HTA) $(7.62 \%)$, enfermedad renal crónica (ERC) $(5.38 \%)$ y diabetes mellitus (DM) (2.02\%) (Tabla 1). 


\section{Discusión}

\section{Resultados principales}

Realizamos un estudio transversal descriptivo para reportar la prevalencia de enfermedades cardíacas y sus comorbilidades en todos los pacientes que fueron admitidos en el servicio de cardiología de un hospital de referencia peruano entre 2016 y 2017. Las enfermedades más frecuentes fueron falla cardíaca, fibrilación auricular e infarto miocárdico. Las comorbilidades de importancia fueron HTA, ERC y DM.

Encontramos una proporción considerable de adultos mayores en el estudio. La edad es un factor de riesgo de diversas enfermedades cardiovasculares ${ }^{15}$. Estudios longitudinales han evidenciado esta relación ${ }^{16,17}$. Cuando el sistema cardiovascular envejece, se deteriora la función endotelial, generando un ambiente proinflamatorio ${ }^{18}$, lo que, consecuentemente, lleva al deterioro de la propia función cardíaca. Por ejemplo, un estudio de 3.140 adultos admitidos en un hospital de Camerún reportó que, a medida que la edad aumentó, la prevalencia de falla cardíaca, accidente cerebrovascular y arritmias también aumentó, siendo más frecuentes en adultos mayores de 70 años ${ }^{19}$. Asimismo, observamos una mayor proporción de hombres que de mujeres en nuestro estudio. Esto concuerda con estudios previos que reportaron la prevalencia de enfermedades cardíacas en hospitales 8,9,19,20. La fisiología de las mujeres no menopáusicas incluye factores hormonales protectores, además se ha sugerido que los hombres están más expuestos a hábitos de vida de riesgo que las mujeres, como tabaquismo, alcoholismo y menor actividad física ${ }^{21,22}$.

Encontramos que más de la mitad de los pacientes sufrieron falla cardíaca. Otras enfermedades importantes fueron fibrilación auricular e infarto miocárdico. La falla cardíaca es una de las enfermedades cardíacas más frecuentes e importantes en el mundo según reportes en EE.UU., Portugal y España ${ }^{23}$. Un metaanálisis reportó que la prevalencia de falla cardíaca en Latinoamérica es del $1 \%{ }^{2}$. Además, la Global Burden of Disease (2017) reportó que la falla cardíaca y la angina relacionada con la enfermedad coronaria fueron causas cardiovasculares importantes de años vividos con discapacidad ${ }^{1}$. Esto podría explicar la frecuencia de falla cardíaca en hospitalizados debido a que su progresión afecta diversos dominios de la independencia funcional, llevando a la discapacidad ${ }^{24}$, hasta que la persona sufre una descompensación. No obstante, la prevalencia varía según el contexto donde se evalúa. Por ejemplo, un estudio en pacientes ingleses reportó que la prevalencia de falla cardíaca fue del $1.1 \%$ en el nivel primario ${ }^{25}$. La diferencia con nuestro estudio podría explicarse porque las causas de consulta en el nivel primario son diferentes a las de emergencias $u$ hospitalización, de esa forma, es más probable encontrar un paciente con falla cardíaca descompensada en una sala de hospitalización que en atención primaria. Siguiendo esta hipótesis, Kaspar, et al. ${ }^{26}$ reportaron una prevalencia de hasta el $55 \%$ en un hospital de Alemania, cifra similar a la nuestra. Un estudio en un centro de atención para adultos mayores reportó que la incidencia acumulada de falla cardíaca fue del $27 \%{ }^{10}$. Zuni-Chavez, et al. ${ }^{27}$ realizaron un muestreo aleatorio de pacientes hospitalizados, y reportaron que la prevalencia de falla cardíaca (26.3\%) lideró las enfermedades cardiovasculares. Por otro lado, el infarto miocárdico agudo y la fibrilación auricular presentan pródromos y manifestaciones, como angina de pecho y síncope, que siempre impulsarán al paciente y/o familiares a acudir a una consulta 0 a emergencias. A pesar de la imperativa necesidad de hospitalización para manejar estas enfermedades, la falla cardíaca es más frecuente y más letal ${ }^{19}$. Por ejemplo, dos estudios en un hospital de referencia peruano evidenciaron que la frecuencia de infartos fue notablemente menor que la de falla cardíaca aguda (175 vs. 1705) ${ }^{8,9}$. Frente a nuestros resultados y a los resultados de estos dos estudios, se debería dar prioridad a la adquisición de recursos para el manejo de estas enfermedades, tanto en emergencias como en hospitalización.

Reportamos que las comorbilidades más frecuentes fueron HTA, ERC y DM. Esto concuerda con estudios previos. Hinton, et al. ${ }^{25}$ estudiaron las enfermedades de 1.275.174 pacientes del Royal College of General Practitioners Research and Surveillance Centre, que es una gran red inglesa de atención primaria. Reportaron que la HTA no controlada, ERC y DM fueron los factores cardiovasculares más comunes, con prevalencias de 17.1, 5.6 y $0.4 \%$, respectivamente. Como se mencionó en el anterior párrafo, la diferencia de contexto con nuestro estudio (atención primaria vs. hospitalario) explica la diferencia de frecuencia de enfermedades cardíacas, sin embargo, las similitud de comorbilidades puede explicarse por la alta prevalencia de estas mundialmente. Por ejemplo, estimados globales sugieren que la prevalencia de HTA en adultos es del $31.1 \%$, siendo uno de los factores cardiovasculares más frecuentes. De esa forma, un estudio de representatividad nacional en Perú reportó una prevalencia del $27.3 \%$ en $2010^{29}$. Además, en Perú, la prevalencia nacional de DM autorreportada es del $3.6 \%{ }^{6}$. Estas cifras representan 
una realidad preocupante que explica las frecuencias altas de ingresos hospitalarios por causas cardíacas, ya que son factores de riesgo importantes. Incluso, esto se ha evidenciado a través de estudios hospitalarios. Ríos Navarro, et al. ${ }^{8}$ realizaron un estudio descriptivo de pacientes con infarto miocárdico agudo de un hospital de referencia peruano, reportando que la prevalencia de HTA, DM y ERC fue del, 58.2, 29.7 y $12 \%$, respectivamente. De la misma forma, Pariona, et al. ${ }^{9}$ realizaron otro estudio descriptivo en pacientes con falla cardíaca aguda, y reportaron que las prevalencias de HTA, DM y ERC fueron 52.6, 29.2 y $30 \%$, respectivamente. El orden de frecuencia de estas cifras concuerda con las nuestras, por lo que los tomadores de decisiones en políticas de salud podrían rescatar esta experiencia para implementar mejores estrategias de prevención primaria de estas comorbilidades de riesgo.

\section{Limitaciones}

Este estudio tiene diversas limitaciones. Primero, al ser un estudio de un solo servicio de cardiología, los resultados no son extrapolables a toda la población peruana. Luego, no recolectamos variables clínicas de cada enfermedad porque el objetivo del estudio fue solo informar de la prevalencia de las enfermedades y sus comorbilidades, no caracterizarlas. Los datos de este estudio fueron recolectados en el periodo de 1 año, lo cual podría introducir un sesgo, ya que la carga de enfermedades puede variar anualmente. Además, el corte transversal de nuestro estudio no permitió evaluar la evolución de las enfermedades. Por ejemplo, un paciente con falla cardíaca compensada pudo progresar a descompensación durante su estancia. Recomendamos futuros estudios de seguimiento de las enfermedades más importantes: falla cardíaca, fibrilación auricular e infarto miocárdico, especialmente para conocer los factores pronósticos. A pesar de estas limitaciones, existen pocos estudios en Perú que hayan revisado exhaustivamente las enfermedades cardíacas en un servicio hospitalario; nuestros resultados serán útiles para el planteamiento de la muestra de otros estudios en enfermedades cardíacas, además esta información podrá ser aprovechable por los administradores en salud, ya que tendrán evidencia para priorizar los recursos para las enfermedades más frecuentes.

\section{Conclusión}

Este estudio reporta las enfermedades y comorbilidades cardiovasculares en un servicio de cardiología de un hospital de referencia peruano. Los resultados servirán para el planteamiento de la muestra de otros estudios, y podrían ser de utilidad para los administradores en salud.

\section{Agradecimientos}

Diego Chambergo-Michilot agradece a María C. por su constante apoyo emotivo.

\section{Conflictos de intereses}

Los autores no reportan ningún conflicto de intereses.

\section{Financiamiento}

Este estudio fue autofinanciado.

\section{Responsabilidades éticas}

Protección de personas y animales. Los autores declaran que para esta investigación no se han realizado experimentos en seres humanos ni en animales.

Confidencialidad de los datos. Los autores declaran que han seguido los protocolos de su centro de trabajo sobre la publicación de datos de pacientes.

Derecho a la privacidad y consentimiento informado. Los autores declaran que en este artículo no aparecen datos de pacientes.

\section{Bibliografía}

1. GBD 2017 Disease and Injury Incidence and Prevalence Collaborators. Global, regional, and national incidence, prevalence, and years lived with disability for 354 diseases and injuries for 195 countries and territories, 1990-2017: a systematic analysis for the Global Burden of Disease Study 2017. Lancet. 2018;392(10159):1789-858.

2. Roth GA, Johnson C, Abajobir A, Abd-Allah F, Abera SF, Abyu G, et al. Global, Regional, and National Burden of Cardiovascular Diseases for 10 Causes, 1990 to 2015. J Am Coll Cardiol. 2017;70(1):1-25.

3. Rubinstein A, Colantonio L, Bardach A, Caporale J, Martí SG, Kopitowski K, et al. Estimation of the burden of cardiovascular disease attributable to modifiable risk factors and cost-effectiveness analysis of preventative interventions to reduce this burden in Argentina. BMC Public Health. 2010;10:627.

4. Dávila-Cervantes CA. Cardiovascular disease in Mexico 1990-2017: secondary data analysis from the global burden of disease study. Int $\mathrm{J}$ Public Health. 2020.

5. Finegold JA, Asaria P, Francis DP. Mortality from ischaemic heart disease by country, region, and age: Statistics from World Health Organisation and United Nations. Int J Cardiol. 2013;168(2):934-45.

6. Instituto Nacional de Estadística e Informática. Perú: Enfermedades No Transmisibles y Transmisibles, 2018 [Internet]. Lima: INEI; 2019 [citado el 13 de julio del 2020]. Disponible en: https://proyectos.inei.gob.pe/endes/2018/SALUD/ENFERMEDADES_ENDES_2018.pdf.

7. Benziger CP, Zavala-Loayza JA, Bernabe-Ortiz A, Gilman $R H$, Checkley W, Smeeth L, et al. Low prevalence of ideal cardiovascular health in Peru. Heart. 2018;104(15):1251-6.

8. Navarro PR, Pariona M, Calderón JAU, Silva FJM. Características clínicas y epidemiológicas del infarto de miocardio agudo en un hospital peruano de referencia. Rev Peru Med Exp y Salud Pública. 2020;37(1):74-80. 
9. Pariona M, Segura Saldaña PA, Padilla Reyes M, Reyes Villanes JS, Jáuregui Contreras M, Valenzuela-Rodríguez G. Características clínico-epidemiológicas de la insuficiencia cardíaca aguda en un hospital terciario de Lima, Perú. Rev Peru Med Exp y Salud Publica. 2017;34(4):655-9.

10. Aronow WS, Ahn C, Gutstein H. Prevalence and incidence of cardiovascular disease in 1160 older men and 2464 older women in a long-term health care facility. J Gerontol A Biol Sci Med Sci. 2002;57(1):M45-46.

11. Ministerio de Salud. Estudio de vulnerabilidad sísmica: estructural, no estructural y funcional del Hospital Nacional Dos de Mayo, el cercado de Lima [Internet]. Lima: MINSA; 2013[citado el 13 de julio del 2020]. Disponible en: http:// sigrid.cenepred.gob.pe/docs/PARA\%20PUBLICAR/CISMID/ESTUDIO\%20 DE\%20VULNERABILIDAD\%20SISMICA\%20ESTRUCTURAL,\%20NO\%20 ESTRUCTURAL\%2OY\%2OFUNCIONAL\%2OHOSPITAL\%2ONACIONAL\%20 DOS\%20DE\%20MAYO,\%20EL\%20CERCADO\%20DE\%20LIMA.PDF.

12. Ministerio de Salud. Hospital Nacional Dos de Mayo. Análisis de la Situación de Salud Hospitalaria [Internet]. Lima: MINSA; 2017 [citado el 13 de julio del 2020]. Disponible en: http://nuevaweb.hdosdemayo.gob.pe/ instrumentos de gestion/normas emitidas/r dir/2018/08 agosto/ RD_0138_ASHO_2017.pdf.

13. Chamberlain JJ, Rhinehart AS, Shaefer CF, Neuman A. Diagnosis and Management of Diabetes: Synopsis of the 2016 American Diabetes Association Standards of Medical Care in Diabetes. Ann Intern Med. 2016;164(8):542-52.

14. Ponikowski P, Voors AA, Anker SD, Bueno H, Cleland JGF, Coats AJS, et al. 2016 ESC Guidelines for the diagnosis and treatment of acute and chronic heart failureThe Task Force for the diagnosis and treatment of acute and chronic heart failure of the European Society of Cardiology (ESC)Developed with the special contribution of the Heart Failure Association (HFA) of the ESC. Eur Heart J. 2016;37(27):2129-200.

15. Rodgers JL, Jones J, Bolleddu SI, Vanthenapalli S, Rodgers LE, Shah K, et al. Cardiovascular Risks Associated with Gender and Aging. J Cardiovasc Dev Dis. 2019;6(2):19.

16. Wang W, Lee ET, Fabsitz RR, Devereux R, Best L, Welty TK, et al. A longitudinal study of hypertension risk factors and their relation to cardiovascular disease: the Strong Heart Study. Hypertension. 2006;47(3):403-9

17. Kelly-Hayes M. Influence of Age and Health Behaviors on Stroke Risk: Lessons from Longitudinal Studies. J Am Geriatr Soc. 2010;58(Suppl 2):S325-8.

18. Donato AJ, Morgan RG, Walker AE, Lesniewski LA. Cellular and Molecular Biology of Aging Endothelial Cells. J Mol Cell Cardiol. 2015;89(0 0):122-35.
19. Nkoke C, Jingi AM, Makoge C, Teuwafeu D, Nkouonlack C, Dzudie A Epidemiology of cardiovascular diseases related admissions in a referral hospital in the South West region of Cameroon: A cross-sectional study in sub-Saharan Africa. PLOS ONE. 2019;14(12):e0226644.

20. Sánchez-Arias AG, Bobadilla-Serrano ME, Dimas-Altamirano B, Gómez-Ortega M, González-González G. Enfermedad cardiovascular: primera causa de morbilidad en un hospital de tercer nivel. Rev Mex Cardiol. 2016;27(S3):98-102

21. Yue Y, Hong L, Guo L, Gao X, Deng J, Huang J, et al. Gender differences in the association between cigarette smoking, alcohol consumption and depressive symptoms: a cross-sectional study among Chinese adolescents. Sci Rep. 2015;5:17959.

22. Craft BB, Carroll HA, Lustyk MKB. Gender Differences in Exercise Habits and Quality of Life Reports: Assessing the Moderating Effects of Reasons for Exercise. Int J Lib Arts Soc Sci. 2014;2(5):65-76.

23. Savarese G, Lund LH. Global Public Health Burden of Heart Failure. Card Fail Rev. 2017;3(1):7-11.

24. Chaudhry SI, McAvay G, Ning Y, Allore HG, Newman AB, Gill TM. Risk Factors for Onset of Disability Among Older Persons Newly Diagnosed with Heart Failure: The Cardiovascular Health Study. J Card Fail. 2011; 17(9):764.

25. Hinton W, McGovern A, Coyle R, Han TS, Sharma P, Correa A, et al. Incidence and prevalence of cardiovascular disease in English primary care: a cross-sectional and follow-up study of the Royal College of General Practitioners (RCGP) Research and Surveillance Centre (RSC). BMJ Open. 2018;8(8):e020282.

26. Kaspar M, Fette G, Güder G, Seidlmayer L, Ertl M, Dietrich G, et al. Underestimated prevalence of heart failure in hospital inpatients: a comparison of ICD codes and discharge letter information. Clin Res Cardiol. 2018;107(9):778-87.

27. Zuni-Chavez KX, More-Sandoval BE, Fernández-Vargas CD, García-Fuentes BB, Ruiz-Olano JM, Pérez-Rodriguez VK. Prevalencia de factores de riesgo cardiovascular en pacientes hospitalizados en un hospital de Lima. Rev Fac Med Hum. 2019;19(4):68-73.

28. Mills KT, Stefanescu A, He J. The global epidemiology of hypertension. Nat Rev Nephrol. 2020;16(4):223-37.

29. Segura-Vega L, Agustí R, Ruiz-Mori E. La Hipertensión Arterial en el Perú según el estudio TORNASOL II. Rev Peru Cardiol. 2011;37(1):19-27. 